\title{
Strong anisotropic optical conductivity in two-dimensional puckered structures: The role of the Rashba effect
}

\author{
S. Saberi-Pouya, ${ }^{1,2}$ T. Vazifehshenas,,${ }^{1, *}$ T. Salavati-fard, ${ }^{3}$ M. Farmanbar, ${ }^{4}$ and F. M. Peeters ${ }^{2}$ \\ ${ }^{1}$ Department of Physics, Shahid Beheshti University, G. C., Evin, Tehran 1983969411, Iran \\ ${ }^{2}$ Department of Physics, University of Antwerp, Groenenborgerlaan 171, B-2020 Antwerpen, Belgium \\ ${ }^{3}$ Department of Physics and Astronomy, University of Delaware, Newark, Delaware 19716, USA \\ ${ }^{4}$ Faculty of Science and Technology and MESA ${ }^{+}$Institute for Nanotechnology, University of Twente, P.O. Box 217, \\ 7500 AE Enschede, The Netherlands
}

(Received 19 April 2017; revised manuscript received 25 June 2017; published 8 August 2017)

\begin{abstract}
We calculate the optical conductivity of an anisotropic two-dimensional system with Rashba spin-flip excitation within the Kubo formalism. We show that the anisotropic Rashba effect caused by an external field significantly changes the magnitude of the spin splitting. Furthermore, we obtain an analytical expression for the longitudinal optical conductivity associated with interband transitions as a function of the frequency for arbitrary polarization angle. We find that the diagonal components of the optical conductivity tensor are direction dependent and the optical absorption spectrum exhibits a strongly anisotropic absorption window. The height and width of this absorption window are very sensitive to the anisotropy of the system. While the height of absorption peak increases with increasing effective mass anisotropy ratio, the peak intensity is larger when the light polarization is along the armchair direction. Moreover, the absorption peak width becomes broader as the density-of-states mass or Rashba interaction is enhanced. These features in the optical absorption spectrum can be used to determine parameters relevant for spintronics.
\end{abstract}

DOI: 10.1103/PhysRevB.96.075411

\section{INTRODUCTION}

Phosphorene, a monolayer of black phosphorus (BP), with puckered structure has attracted considerable attention because of the unique physical properties associated with its anisotropic band structure [1-6]. BP is a layered material in which each layer forms a puckered surface due to $s p^{3}$ hybridization. In its bulk crystalline form, BP is a semiconductor with a direct band gap of $0.3 \mathrm{eV}$ which reaches up to $2 \mathrm{eV}$ in the monolayer structure $[7,8]$. Phosphorenelike materials, group IV-VI compounds, resemble in many respects, for instance, inplane anisotropy, orthorhombic lattice, and puckered layered structure [9-11]. Similar to phosphorene and as a consequence of their orthorhombic structure, transport, optoelectronic, and spintronic properties of these materials are highly anisotropic $[12,13]$.

Nowadays, spin-orbit coupling interaction is a field of great interest owing to potential applications in spintronic phenomena and electric manipulation of spins [14-20]. This interaction shows up when crystalline lattices lack a center of inversion symmetry (the Dresselhaus type [21]) or there is a structural asymmetry in the interface/surface region of quantum wells (the Rashba type [22]). In two-dimensional (2D) materials, when inversion symmetry is broken by an applied transverse electric field or a substrate, spin degeneracy is lifted due to the Rashba effect [23-26]. Therefore, transitions between spin-split states results in a nonzero value for the optical conductivity in the presence of an alternating electric field. The absorption part of the optical conductivity can be used in order to probe the spin-split energy levels. Like the unique anisotropic properties of excitons in phosphorene [27], the spintronic parameters such as the Rashba coupling

\footnotetext{
*t-vazifeh@sbu.ac.ir
}

strength, electron density, and also spin polarization in the 2D materials can be measured optically [28-34].

Recently, Xiao et al. [31] studied the optical conductivity of $\mathrm{MoS}_{2}$ in the presence of spin-orbit coupling and found that the Rashba spin-orbit parameter can change the absorption peak or absorption window in the optical spectrum. In contrast to the isotropic band structure of $\mathrm{MoS}_{2}$, the anisotropic band structure in phosphorene results in a highly anisotropic Rashba splitting [35,36]; hence, the strength of spin splitting depends on the direction of wave vector, as well as its magnitude [36]. The anisotropic Rashba spin-orbit interaction in 2D electron or hole gas systems due to the $k$-cubic Rashba spin-orbit interaction gives rise to different features in the optical conductivity, anisotropic spin susceptibility, and also distinctive behavior of the spin Hall conductivity [37,38]. Another anisotropic behavior of the spin splitting appears for the interplay between both Rashba and Dresselhaus spinorbit coupling in a $2 \mathrm{D}$ electron gas. It has been shown that the anisotropic dynamical optical conductivity can be used as a powerful tool to probe and manipulate the coupling strengths and set out the range of frequency where the optical conductivity is essentially nonzero $[34,39]$.

In this paper, we use the $k$-linear Rashba Hamiltonian for anisotropic 2D materials, such as phosphorene and group-IV monochalcogenides, which have been predicted to exhibit an anisotropic energy band [10,12,40,41]. Based upon the Kubo formalism, we obtain an analytical expression for the longitudinal optical conductivity with arbitrary polarization angles, including interband transitions. In contrast to isotropic systems, the longitudinal optical conductivity depends not only on the Rashba parameter, but most importantly also on the effective mass anisotropy. The most significant contribution to the optical absorption occurs when the polarization of light is along the armchair direction (the direction with a smaller effective mass). We find that the extrinsic spin-orbit coupling 
due to the broken inversion symmetry is strongly anisotropic, which has an impact on the optical response of 2D electron systems. The level splitting caused by the anisotropic Rashba effect gives rise to an optical response with a directiondependent absorption peak. The width of the absorption window strongly depends on both the polarization direction and the effective mass ratio. The latter can be considered as an additional degree of freedom to tune the height and width of this absorption peak. High carrier mobility or increased electric field shifts the position of the absorptive peak to higher frequencies, which can be adjusted appropriately. Our calculations of the optical conductivity with Rashba spin-orbit coupling are compared with measurements of infrared spectra on BP thin films for different polarizations [41]. Moreover, these values compare favorably with the optical conductivity of $\mathrm{MoS}_{2}$, a typical 2D material with nonpuckered structure, in the presence of Rashba interaction at low temperature in the infrared to terahertz $(\mathrm{THz})$ spectral range [31]. We predict that single- and few-layer BP similar to some other 2D materials (e.g., $\mathrm{MoS}_{2}$ ) have a great potential for infrared optoelectronics. Whereas phosphorene is a good example of a $2 \mathrm{D}$ anisotropic system, our formalism is also applicable to other 2D puckered materials.

Our paper is organized as follows. In Sec. II, we describe the basic Hamiltonian used in this work in the presence of anisotropic Rashba effect and develop a general formalism applicable for anisotropic 2D materials. We obtain in Sec. III an analytical expression for the optical conductivity due to spinflip transitions. We present our numerical results and provide a discussion of our findings. The highlights of this work are summarized in Sec. IV.

\section{PARABOLOIDAL ENERGY BAND WITH ANISOTROPIC RASHBA EFFECT}

We study the low-energy dispersion and optical conductivity of a 2D system with paraboloidal energy band in the presence of Rashba spin-orbit coupling. We assume that the 2D system is a puckered honeycomb lattice where the $x$ and $y$ axes are taken to be along its armchair and zigzag directions, respectively. The Hamiltonian for such a system including the extrinsic Rashba term is given by

$$
\hat{H}=\hat{H}_{0}+\hat{H}_{R} \text {. }
$$

Here, $\hat{H}_{0}$ is the $\mathbf{k} \cdot \mathbf{p}$ free-electron Hamiltonian whose lowenergy spectrum for an anisotropic $2 \mathrm{D}$ system is obtained as [42]

$$
\hat{H}_{0}=\frac{\hbar^{2}}{2}\left(\frac{k_{x}^{2}}{m_{x}}+\frac{k_{y}^{2}}{m_{y}}\right) \hat{\sigma_{0}},
$$

where $m_{x}$ and $m_{y}$ are the electron effective masses along the $x$ and $y$ directions, respectively, and $\hat{\sigma_{0}}$ is a $2 \times 2$ unitary matrix. The Rashba anisotropic Hamiltonian, which has been recently introduced for phosphorene [36], can be rewritten as

$$
\hat{H}_{R}=-\alpha_{R}\left(\sqrt{\frac{m_{d}}{m_{x}}} k_{x} \hat{\sigma_{y}}-\sqrt{\frac{m_{d}}{m_{y}}} k_{y} \hat{\sigma_{x}}\right) .
$$

Here, $\alpha_{R}$ is the Rashba coefficient, $m_{d}=\sqrt{m_{x} m_{y}}$ is the density-of-states mass, and $\hat{\sigma_{x}}$ and $\hat{\sigma_{y}}$ are the Pauli matrices.

Upon diagonalizing the total Hamiltonian, one obtains two branches of the energy spectrum:

$$
E^{\lambda}(\mathbf{k})=\frac{\hbar^{2} k^{2} R(\theta)}{2}+\lambda \alpha_{R}^{*}(\theta) k,
$$

where $\lambda$ refers to the electron spin in the upper $(+)$ or lower $(-)$ branch, and $\theta$ denotes the angle of the wave vector with respect to the $x$ axis. $R(\theta)$, the orientation parameter, is defined as [43]

$$
R(\theta)=\left(\frac{\cos ^{2} \theta}{m_{x}}+\frac{\sin ^{2} \theta}{m_{y}}\right),
$$

and $\alpha_{R}^{*}$ is the anisotropic Rashba coefficient, given by

$$
\alpha_{R}^{*}(\theta)=\alpha_{R} \sqrt{m_{d} R(\theta)} .
$$

To illustrate the effect of Rashba spin-orbit coupling on the anisotropic band structure, we plot the energy dispersion of the Rashba spin-split branches and energy spacing (the energy difference between the spin-up and spin-down branches) along the two main crystallographic directions in Fig. 1. Due to the Rashba interaction, the energy dispersion deviates from a parabola for each spin branch. Moreover, the anisotropic characteristic of the spin-split branches as a result of different effective masses along the armchair $(x)$ and zigzag $(y)$
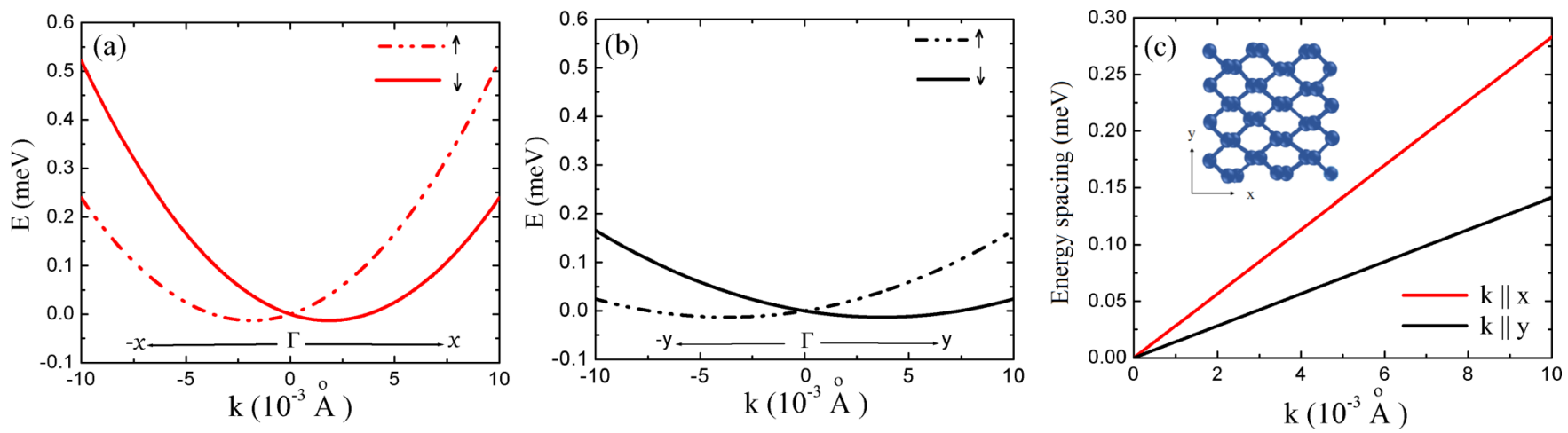

FIG. 1. Energy dispersion (a) along the $x$ direction $(\theta=0)$ and (b) along the $y$ direction $(\theta=\pi / 2)$ with $m_{x}=m_{0}, m_{y}=4 m_{0}$, and $\alpha_{R}=10 \mathrm{meV} \AA$. (c) Spin splitting along the $x$ and $y$ directions for several values of $\alpha_{R}$ with $m_{x}=m_{0}$ and $m_{y}=4 m_{0}$. The inset figure is a top view of an anisotropic monolayer with the $x$ and $y$ axes being the armchair and zigzag directions, respectively. 

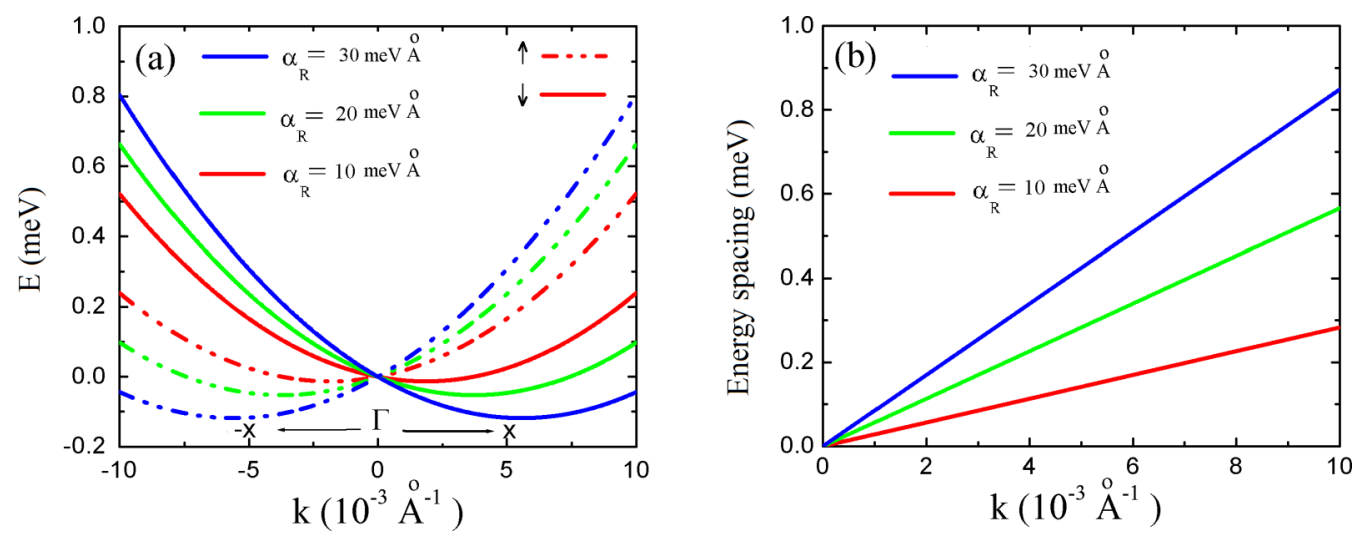

FIG. 2. (a) Energy dispersion and (b) spin splitting along the $x$ (armchair) direction for a few values of $\alpha_{R}$ with $m_{y}=4 m_{0}$ and $m_{x}=m_{0}$.

directions is clearly observed. Also, it can be seen from Fig. 1(c) that the energy is direction dependent and, as expected, along the armchair direction with smaller effective mass, the spin splitting is stronger. It is known that the Rashba spin-orbit interaction can be tuned by an external electric field, so we show the energy dispersion and energy spacing along the armchair $(x)$ direction for different Rashba parameters in Figs. 2(a) and 2(b). We find that the dispersion relation of a $2 \mathrm{D}$ material with anisotropic band structure can be well tuned by the directional effective masses as well as the Rashba coefficient. Besides, there is a uniform enhancement of the energy spacing with increasing Rashba parameter due to the linear momentum dependence of the spin-splitting interaction.

\section{OPTICAL CONDUCTIVITY}

In a spin-orbit coupled system, the optical conductivity due to the transitions between different spin states is an important quantity. We calculate this property using the Kubo linear response formalism for a $2 \mathrm{D}$ system with anisotropic parabolic energy band in the presence of Rashba interaction. Assuming a spatially homogeneous electric field, the Kubo formula for conductivity which starts from the current-current correlation function is given by $[22,44]$

$$
\begin{aligned}
\sigma_{i j}(\mathbf{q}, t)= & \frac{i n e^{2}}{m \omega} \delta_{i j} \\
& +\frac{1}{\omega} \int_{0}^{\infty} e^{i \bar{\omega}}\left\langle\left[\hat{J}_{i}(\mathbf{q}, t), \hat{J}_{j}(\mathbf{q}, 0)\right]\right\rangle d t
\end{aligned}
$$

where indices $i$ and $j$ stand for the two Cartesian coordinates $x, y, n$ is the electron density, $\bar{\omega}=\omega+i \eta\left(\eta \rightarrow 0^{+}\right)$, and $\hat{J}_{i}=$ $e \hat{v}_{i}$ is the current density operator with $\hat{v}_{i}=\hbar^{-1} \partial \hat{H} / \partial k_{i}$ being the electron velocity operator. In this paper, we concentrate on the absorptive part of the optical conductivity which is the real part of this complex quantity [45]. In the optical limit $q \rightarrow 0$, the dynamical optical conductivity can be obtained as follows:

$$
\begin{aligned}
\sigma_{i j}(\omega)= & \frac{i e^{2}}{\omega} \sum_{\lambda, \lambda^{\prime}} \sum_{\mathbf{k}, \mathbf{k}^{\prime}} \psi_{i j}\left(\mathbf{k}, \mathbf{k}^{\prime}, \lambda, \lambda^{\prime}\right) \\
& \times \frac{f^{0}\left(E^{\lambda}(\mathbf{k})\right)-f^{0}\left(E^{\lambda^{\prime}}\left(\mathbf{k}^{\prime}\right)\right)}{E^{\lambda}(\mathbf{k})-E^{\lambda^{\prime}}\left(\mathbf{k}^{\prime}\right)+\hbar(\omega+i \eta)},
\end{aligned}
$$

where $\psi_{i j}\left(\mathbf{k}, \mathbf{k}^{\prime}, \lambda, \lambda^{\prime}\right)$ is defined as

$$
\psi_{i j}\left(\mathbf{k}, \mathbf{k}^{\prime}, \lambda, \lambda^{\prime}\right)=\left\langle\mathbf{k} \lambda\left|\hat{v}_{i}\right| \mathbf{k}^{\prime} \lambda^{\prime}\right\rangle>\left\langle\mathbf{k}^{\prime} \lambda^{\prime}\left|\hat{v}_{j}\right| \mathbf{k} \lambda\right\rangle .
$$

The electron velocity operators for two spin-split branches in the $x$ and $y$ directions are given as

$$
\begin{aligned}
& \hat{v}_{x}=\frac{1}{\hbar}\left(\frac{\hbar^{2} k_{x}}{m_{x}} \hat{\sigma}_{0}-\alpha_{R} \sqrt{\frac{m_{d}}{m_{x}}} k_{x} \hat{\sigma}_{y}\right), \\
& \hat{v}_{y}=\frac{1}{\hbar}\left(\frac{\hbar^{2} k_{y}}{m_{y}} \hat{\sigma}_{0}+\alpha_{R} \sqrt{\frac{m_{d}}{m_{y}}} k_{y} \hat{\sigma}_{x}\right) .
\end{aligned}
$$

Before calculating the eigenstates of the system, $|\mathbf{k} \lambda\rangle$, we introduce a new 2D wave vector $\mathbf{p}=\left(p_{x}, p_{y}\right)$, which is defined as $\mathbf{k}=\sqrt{M / m_{d}} \mathbf{p}$ with $M$ being the mass tensor whose diagonal elements are $m_{x}$ and $m_{y}$ [46]. Now, we can rewrite the free electron and Rashba parts of the total Hamiltonian as follows:

$$
\begin{aligned}
\hat{H}_{0} & =\frac{\hbar^{2} p^{2}}{2 m_{d}} \hat{\sigma}_{0}, \\
\hat{H}_{R} & =-\alpha_{R}(\mathbf{p} \times \hat{z}) \cdot \hat{\sigma},
\end{aligned}
$$

where $\hat{\sigma}=\hat{\sigma}_{x} \hat{i}+\hat{\sigma}_{y} \hat{j}$. Thus, the two spin-split eigenstates can be identified as

$$
|\mathbf{p} \lambda\rangle=\frac{e^{i \mathbf{p} \cdot \mathbf{r}}}{\sqrt{2}}\left(\begin{array}{c}
1 \\
\lambda \frac{p_{y}-i p_{x}}{p}
\end{array}\right),
$$

with $\mathbf{r}=(x, y)$ being a $2 \mathrm{D}$ real-space position vector. Also, the expressions for the velocity operators can be written in terms of $\mathbf{p}$ :

$$
\begin{aligned}
& \hat{v}_{x}=\frac{\hbar}{\sqrt{m_{x} m_{d}}}\left(p_{x} \hat{\sigma}_{0}-p_{R} \hat{\sigma}_{y}\right), \\
& \hat{v}_{y}=\frac{\hbar}{\sqrt{m_{y} m_{d}}}\left(p_{y} \hat{\sigma}_{0}+p_{R} \hat{\sigma}_{x}\right),
\end{aligned}
$$

where $p_{R}=\alpha_{R} m_{d} / \hbar^{2}$ is the effective Rashba wave vector. It has been shown that the spin-conserving intraband transitions give rise to low-frequency absorption, whereas the spin-flip transitions result in a wide absorption peak [31,34]. We focus on the optical conductivity due to the interband spin-flip excitations. As ordinary 2D electron gas systems [34], it can be shown that the off-diagonal elements of the optical conductivity (transverse or Hall conductivity) are zero in the 
absence of a magnetic field, i.e., $\sigma_{x y}=0$. By making use of Eqs. (13) and (14), one can calculate the diagonal elements of the $\psi$ tensor as

$$
\begin{aligned}
\psi_{j j}\left(\mathbf{p}, \mathbf{p}^{\prime} ; \lambda, \lambda^{\prime}\right)= & \frac{\hbar^{2}}{2 m_{j} m_{d}}\left[p_{j}^{2}\left(1+\lambda \lambda^{\prime}\right)+p_{\alpha}^{2}\left(1+\lambda \lambda^{\prime} \cos 2 \phi\right)\right. \\
& \left.+2 p_{R} p_{j} \cos \phi\left(1+\lambda \lambda^{\prime}\right)\right] \delta_{\mathbf{p} \mathbf{p}^{\prime}}
\end{aligned}
$$

where $\phi=\tan ^{-1}\left(p_{y} / p_{x}\right)$. Accordingly, the nonequal diagonal components of optical conductivity (longitudinal optical conductivity) due to spin-flip transitions are obtained as

$$
\begin{aligned}
\sigma_{j j}(\omega)= & \frac{i e^{2} \hbar^{2} p_{R}}{2 \omega m_{j} m_{d}} \int \frac{p d p d \phi}{(2 \pi)^{2}}(1-\cos 2 \phi) \\
& \times\left[\frac{f^{0}\left(E^{-}(\mathbf{p})\right)-f^{0}\left(E^{+}(\mathbf{p})\right)}{E^{-}(\mathbf{p})-E^{+}(\mathbf{p})+\hbar(\omega+i \eta)}+\left(E^{-} \leftrightarrow E^{+}\right)\right] .
\end{aligned}
$$

After performing the $\mathbf{p}$ integral, the following expression is obtained for $\sigma_{j j}$ at zero temperature:

$$
\sigma_{j j}(\omega)=-\frac{i e^{2} m_{d}^{2}}{2 \pi m_{j} \omega \hbar^{4}}\left[1+\frac{\hbar^{3} \omega R(\theta) \Lambda(\omega)}{8\left(\alpha_{R}^{*}(\theta)\right)^{2}}\right],
$$

with $\Lambda(\omega)$ defined as

$$
\Lambda(\omega)=\ln \left[\frac{\left(\omega-\omega_{-}+i \eta\right)\left(\omega+\omega_{+}+i \eta\right)}{\left(\omega+\omega_{-}+i \eta\right)\left(\omega-\omega_{+}+i \eta\right)}\right],
$$

where $\omega_{ \pm}$are the threshold frequency modes induced by the interbranch electronic transition:

$$
\omega_{ \pm}=\frac{2 \alpha_{R}^{*} p_{ \pm}^{F}}{\sqrt{m_{d} R(\theta)} \hbar} .
$$

Here, $p_{ \pm}^{F}=p_{F} \mp p_{R}$ are the Fermi wave vectors for the two spin branches with $p_{F}=\sqrt{2 n \pi-p_{R}^{2}}$. A schematic diagram for the optical transitions is shown in Fig. 3. The

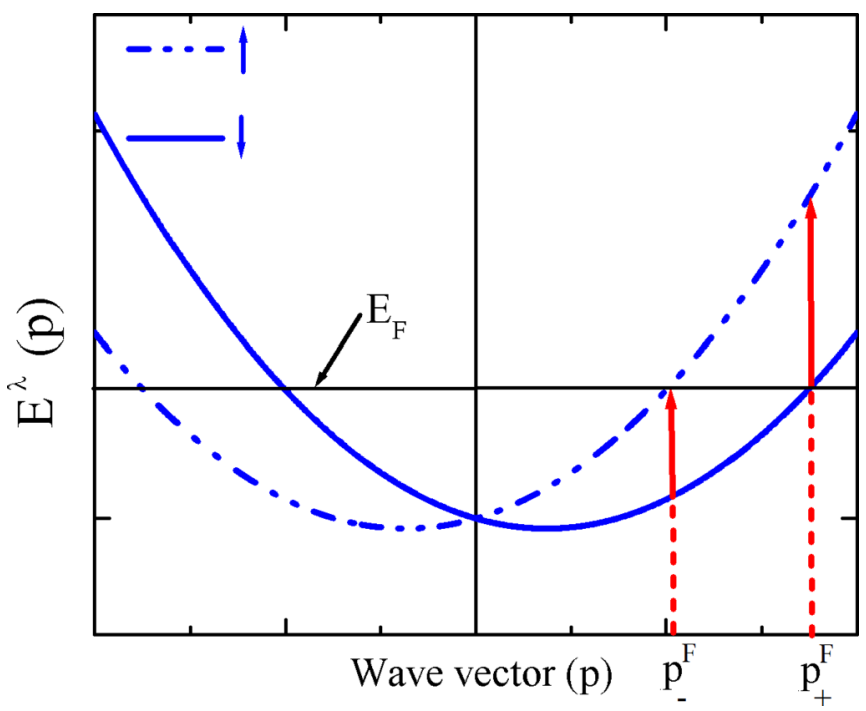

FIG. 3. Scheme of transitions between spin branches. The arrows correspond to the transitions with threshold frequencies of $\left(2 \alpha_{R} p_{F}^{ \pm}\right) / \hbar$.
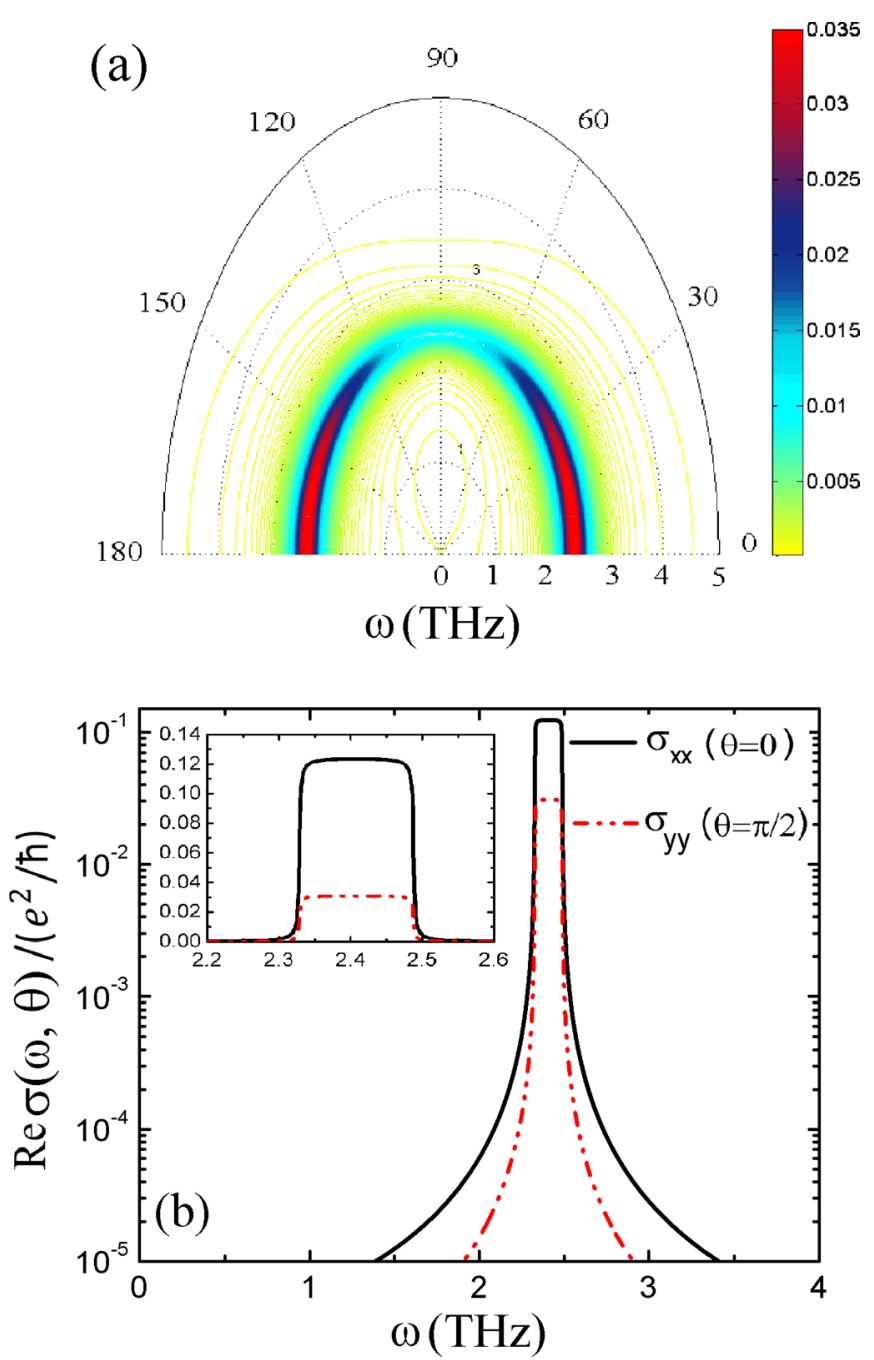

FIG. 4. Real part of longitudinal optical conductivity as a function of excitation frequency: (a) for all $\theta$, and (b) for $\theta=0$ and $\theta=\pi / 2$, with $n=1 \times 10^{13} \mathrm{~cm}^{-2}$. Here we set $\alpha_{R}=10 \mathrm{meV} \AA, m_{y}=4 m_{0}$, and $m_{x}=m_{0}$.

arrows correspond to the vertical transitions between two spin branches in the optical limit $(q \rightarrow 0)$.

The longitudinal optical conductivity along an arbitrary polarization direction $\theta$ is given by [47]

$$
\sigma(\omega, \theta)=\sigma_{x x} \cos ^{2} \theta+\sigma_{y y} \sin ^{2} \theta .
$$

By inserting Eqs. (17) and (18) in the above equation, we obtain

$$
\sigma(\omega, \theta)=-\frac{i e^{2} m_{d}\left(\alpha_{R}^{*}(\theta)\right)^{2}}{2 \pi \omega \hbar^{4}}\left[1+\frac{\hbar^{3} \omega R(\theta) \Lambda(\omega)}{8\left(\alpha_{R}^{*}(\theta)\right)^{2}}\right] .
$$

In Fig. 4(a), we illustrate the calculated real part of the anisotropic conductivity for arbitrary direction of polarization vector as a function of radiation frequency, $\omega$, at a fixed electron density $n=1 \times 10^{13} \mathrm{~cm}^{-2}$ and for $\alpha_{R}=10 \mathrm{meV} \AA, m_{y}=$ $4 m_{0}$, and $m_{x}=m_{0}$. As expected, the optical conductivity has its maximum value at $\theta=0$, i.e., along the armchair direction of the 2D layer. The significantly smaller effective mass for 

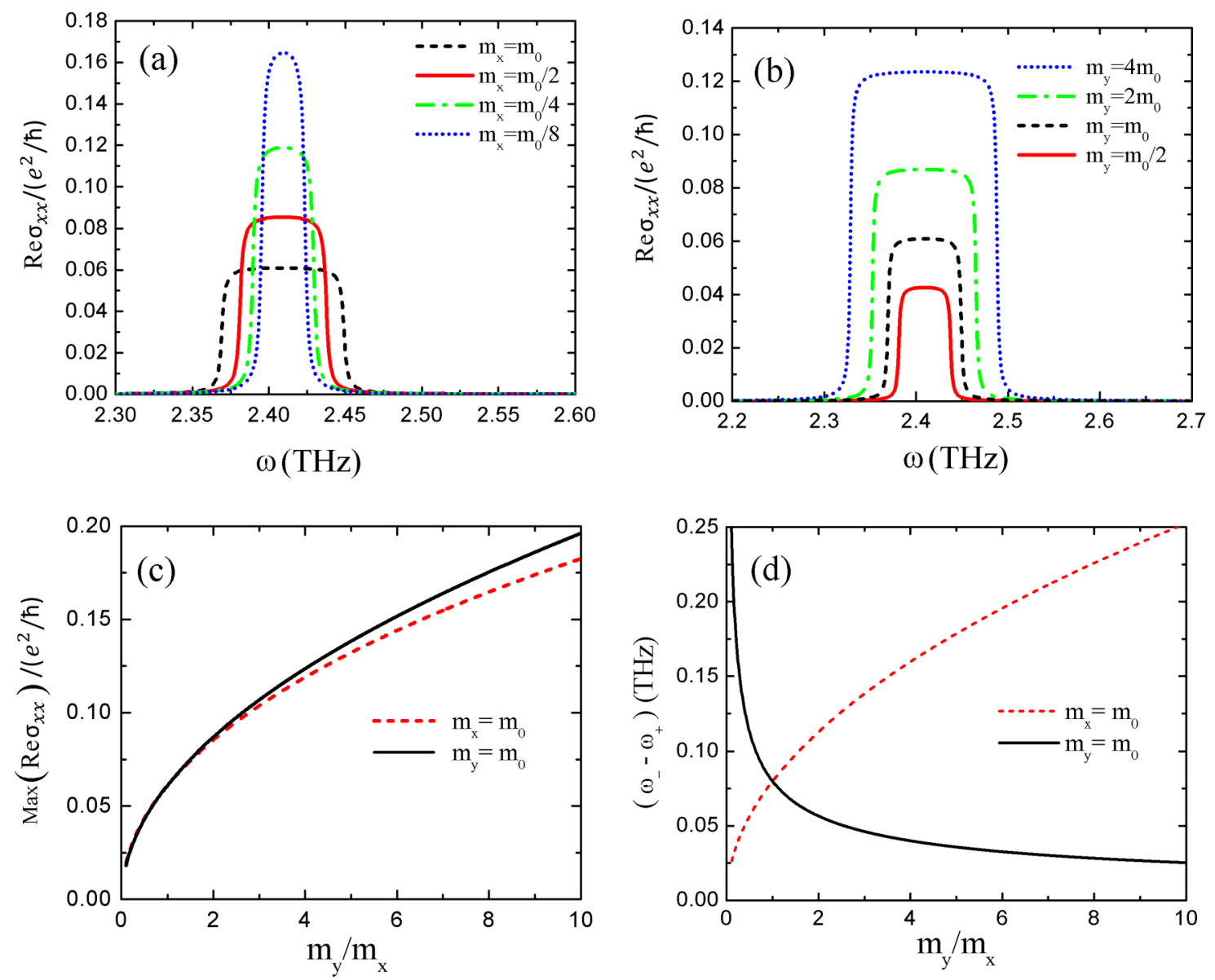

FIG. 5. Real part of longitudinal optical conductivity as a function of excitation frequency for $\theta=0$ with (a) $m_{y}=4 m_{0}$ and (b) $m_{x}=m_{0}$. (c) Maximum of real part of longitudinal optical conductivity as a function of mass anisotropy ratio. (d) Width of the absorption window as a function of mass anisotropy ratio. Here, we set $n=1 \times 10^{13} \mathrm{~cm}^{-2}$ with $\alpha_{R}=10 \mathrm{meV} \AA$.

the $\theta=0$ direction suggests that the charge carriers prefer to flow along this direction. In addition, Fig. 4(b) shows the absorption part of the optical conductivity for the two main crystallographic directions, $\theta=0$ and $\theta=\pi / 2$. The fact that the anisotropy ratio of the optical conductivity is equal to the inverse of the mass anisotropy ratio, i.e., $\sigma_{y y}(\omega) / \sigma_{x x}(\omega)=$ $m_{x} / m_{y}$, is clearly observed in this figure. Furthermore, as a consequence of the energy conservation law under vertical transitions $(q \rightarrow 0)$ between spin branches (see Fig. 3), the absorption part of the longitudinal optical conductivity at $T=0$ has a step-function-like variation with the frequency, i.e., a nonzero value for a range of frequencies which is given by

$$
\omega_{-}-\omega_{+}=\frac{2\left(\alpha_{R}^{*}\right)^{2}}{\hbar^{3} R(\theta)} .
$$

As is evident from Fig. 4, the absorption is in the THz region for the set of parameters used here.

We have already mentioned above that the optical conductivity exhibits a strong dependence on the effective mass anisotropy. The variation of the real part of $\sigma_{x x}$ with the effective mass along the $x$ and $y$ directions is shown in Fig. 5. One may notice that the mass asymmetry not only alters the maximum value of the optical conductivity but also changes the width of the peak. According to this figure, the peak height of $\sigma_{x x}(\omega)$ increases by decreasing the effective mass along the radiation polarization direction or increasing the effective mass along the direction perpendicular to the radiation polarization. In other words, a higher peak intensity is achieved as a consequence of the effective mass anisotropy ratio $\left(m_{y} / m_{x}\right)$ enhancement. Moreover, the absorption window of the real part of longitudinal optical conductivity is extended by increasing the density-of-states mass $\left(m_{d}\right)$.

The effect of Rashba coupling strength on the absorption part of $\sigma_{x x}(\omega)$ is shown in Fig. 6(a). The absorption peak width is broadened and the onset frequency of the absorptive peak moves towards a higher value when the Rashba parameter increases [28]. The Rashba parameters chosen here are comparable to the as-obtained values for phosphorene and group-IV monochalcogenides from density functional theory calculations [35,36]. For large values of $\alpha_{R}$, a stronger spin splitting occurs, which in turn shifts the absorption part of the optical conductivity from the $\mathrm{THz}$ to infrared frequencies. These features for the spin-flip absorption peak are similar to that of the conventional 2D electron gas system and $\mathrm{MoS}_{2}$ [31]. We observe that the measurements of infrared spectra on BP thin films for different polarizations [41] are in good agreement with our results for the absorption part of the optical conductivity at the high values of Rashba parameter and carrier density, especially across the technologically relevant $\mathrm{THz}$ to 

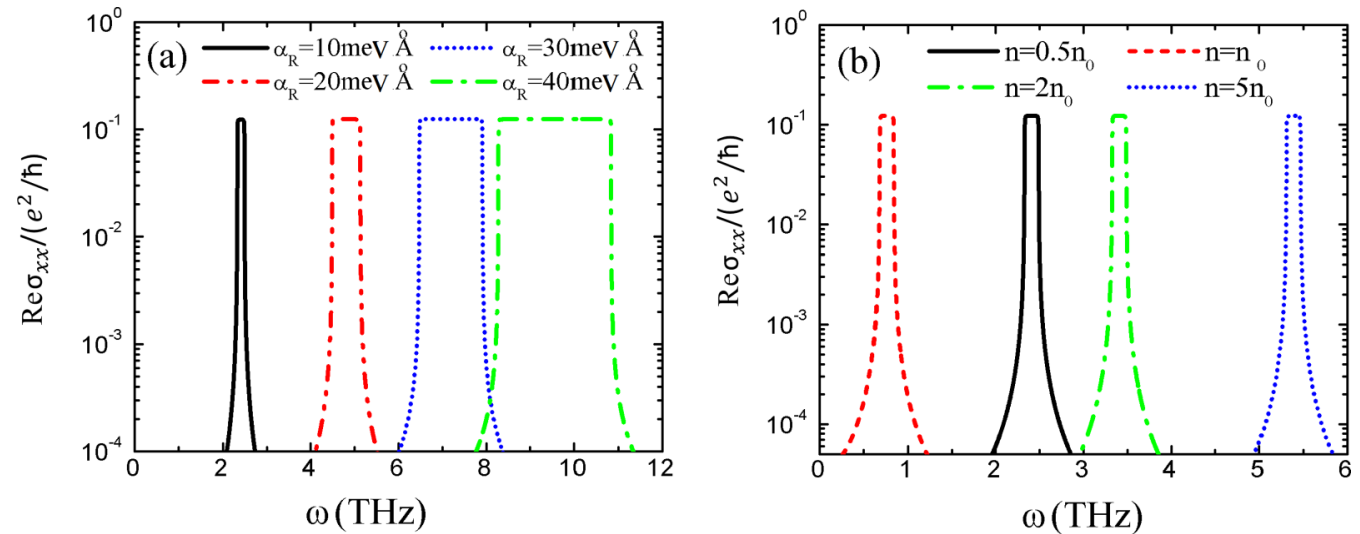

FIG. 6. Real part of longitudinal optical conductivity as a function of excitation frequency for $\theta=0$ with $m_{y}=4 m_{0}, m_{x}=m_{0}$ for a few values of (a) Rashba parameter with $n_{0}=1 \times 10^{13} \mathrm{~cm}^{-2}$ and (b) electron density with $\alpha_{R}=10 \mathrm{meV} \AA$.

midinfrared spectrum [47]. We also depict the variation of the optical absorption with the electron density, in Fig. 6(b), for fixed $\alpha_{R}=10 \mathrm{meV} \AA$ and effective masses $m_{y}=4 m_{0}$ and $m_{x}=m_{0}$. One of the important characteristics of this figure is that while the value of the peak height remains almost unchanged by increasing electron density, the absorption peak moves toward higher frequencies as a result of the Pauli blockade effect [48-50].

\section{CONCLUSIONS}

In summary, we have studied the energy spectrum and optical response of an anisotropic 2D electron gas system in the presence of Rashba spin-orbit interaction. Based on the Kubo formalism, we calculated the optical conductivity tensor considering the Rashba spin-flip excitations. We found that the effective mass anisotropy plays an important role in the optical absorption spectrum through the direction-dependent Rashba spin splitting. As a general result, the diagonal components of the optical conductivity tensor are inversely proportional to the corresponding effective mass elements. Furthermore, the effective mass asymmetry is an additional degree of freedom to tune the height and width of the absorption peak. This introduces aspects to the optical conductivity for spintronic applications of 2D anisotropic materials such as phosphorene and group-IV monochalcogenides. We also showed that larger optical absorption is generated when the polarization of radiation is along the armchair direction and its maximal value is enhanced by increasing the effective mass ratio. However, the width of the absorption window has a strong dependence on both the polarization direction and the effective mass ratio. Finally, the position of the absorptive peak moves to higher frequencies with increasing the Rashba parameter and electron density. Our results suggest an interesting way to determine some of the spintronic characteristics of a class of 2D nanostructures, with anisotropic Rashba effect, using optical methods.
[1] A. Carvalho, M. Wang, X. Zhu, A. S. Rodin, H. Su, and A. H. C. Neto, Nat. Rev. Mater. 1, 16061 (2016).

[2] X. Ling, H. Wang, S. Huang, F. Xia, and M. S. Dresselhaus, Proc. Natl. Acad. Sci. USA 112, 4523 (2015).

[3] E. Taghizadeh Sisakht, F. Fazileh, M. H. Zare, M. Zarenia, and F. M. Peeters, Phys. Rev. B 94, 085417 (2016).

[4] M. Zare, B. Z. Rameshti, F. G. Ghamsari, and R. Asgari, Phys. Rev. B 95, 045422 (2017).

[5] S. Brener, A. N. Rudenko, and M. I. Katsnelson, Phys. Rev. B 95, 041406 (2017).

[6] S. Saberi-Pouya, T. Vazifehshenas, T. Salavati-fard, and M. Farmanbar, arXiv:1705.00472.

[7] V. Tran, R. Soklaski, Y. Liang, and L. Yang, Phys. Rev. B 89, 235319 (2014).

[8] B. Sa, Y.-L. Li, Z. Sun, J. Qi, C. Wen, and B. Wu, Nanotechnology 26, 215205 (2015).

[9] S. Zhang, N. Wang, S. Liu, S. Huang, W. Zhou, B. Cai, M. Xie, Q. Yang, X. Chen, and H. Zeng, Nanotechnology 27, 274001 (2016).

[10] L. C. Gomes and A. Carvalho, Phys. Rev. B 92, 085406 (2015).
[11] C. Kamal, A. Chakrabarti, and M. Ezawa, Phys. Rev. B 93, 125428 (2016).

[12] L. Medrano Sandonas, D. Teich, R. Gutierrez, T. Lorenz, A. Pecchia, G. Seifert, and G. Cuniberti, J. Phys. Chem. C 120, 18841 (2016).

[13] Z. Tian, C. Guo, M. Zhao, R. Li, and J. Xue, ACS Nano 11, 2219 (2017).

[14] I. Žutić, J. Fabian, and S. Das Sarma, Rev. Mod. Phys. 76, 323 (2004).

[15] H. Min, J. E. Hill, N. A. Sinitsyn, B. R. Sahu, L. Kleinman, and A. H. MacDonald, Phys. Rev. B 74, 165310 (2006).

[16] D. Pesin and A. H. MacDonald, Nat. Mater. 11, 409 (2012).

[17] J. Wunderlich, B. Kaestner, J. Sinova, and T. Jungwirth, Phys. Rev. Lett. 94, 047204 (2005).

[18] K. V. Shanavas and S. Satpathy, Phys. Rev. Lett. 112, 086802 (2014).

[19] V. Galitski and I. B. Spielman, Nature 494, 49 (2013).

[20] A. Al-Hilli and B. Evans, J. Cryst. Growth 15, 93 (1972).

[21] G. Dresselhaus, Phys. Rev. 100, 580 (1955). 
[22] E. I. Rashba, Sov. Phys. Solid State 2, 1224 (1960).

[23] C. L. Kane and E. J. Mele, Phys. Rev. Lett. 95, 226801 (2005).

[24] Y. A. Bychkov and E. I. Rashba, J. Phys. C 17, 6039 (1984).

[25] A. Manchon, H. C. Koo, J. Nitta, S. M. Frolov, and R. A. Duine, Nature (London) 14, 871 (2015).

[26] W.-K. Tse and S. Das Sarma, Phys. Rev. B 74, 245309 (2006).

[27] X. Wang, A. M. Jones, K. L. Seyler, V. Tran, Y. Jia, H. Zhao, H. Wang, L. Yang, X. Xu, and F. Xia, Nat. Nanotechnol. 10, 517 (2015).

[28] D. W. Yuan, W. Xu, Z. Zeng, and F. Lu, Phys. Rev. B 72, 033320 (2005).

[29] C. H. Yang, W. Xu, Z. Zeng, F. Lu, and C. Zhang, Phys. Rev. B 74, 075321 (2006).

[30] Y. S. Ang, J. C. Cao, and C. Zhang, Eur. Phys. J. B 87, 28 (2014).

[31] Y. M. Xiao, W. Xu, B. Van Duppen, and F. M. Peeters, Phys. Rev. B 94, 155432 (2016).

[32] Z. Li and J. P. Carbotte, Phys. Rev. B 86, 205425 (2012).

[33] K. F. Mak, C. Lee, J. Hone, J. Shan, and T. F. Heinz, Phys. Rev. Lett. 105, 136805 (2010).

[34] D. Yudin and I. A. Shelykh, Phys. Rev. B 94, 161404 (2016).

[35] M. Kurpas, M. Gmitra, and J. Fabian, Phys. Rev. B 94, 155423 (2016).

[36] Z. S. Popović, J. M. Kurdestany, and S. Satpathy, Phys. Rev. B 92, 035135 (2015).
[37] J. Zhou, W.-Y. Shan, and D. Xiao, Phys. Rev. B 91, 241302 (2015).

[38] A. Mawrie and T. K. Ghosh, J. Phys.: Condens. Matter 28, 425302 (2016).

[39] D. Yudin, D. R. Gulevich, and I. A. Shelykh, Phys. Rev. B 95, 035401 (2017).

[40] T.-H. Liu and C.-C. Chang, Nanoscale 7, 10648 (2015).

[41] F. Xia, H. Wang, and Y. Jia, Nat. Commun. 5, 4458 (2014).

[42] A. S. Rodin, A. Carvalho, and A. H. Castro Neto, Phys. Rev. Lett. 112, 176801 (2014).

[43] S. Saberi-Pouya, T. Vazifehshenas, M. Farmanbar, and T. Salavati-fard, J. Phys.: Condens. Matter 28, 285301 (2016).

[44] L. Magarill, A. Chaplik, and M. Éntin, J. Exp. Theor. Phys. 92, 153 (2001).

[45] A. Bácsi and A. Virosztek, Phys. Rev. B 87, 125425 (2013).

[46] T. Low, R. Roldán, H. Wang, F. Xia, P. Avouris, L. M. Moreno, and F. Guinea, Phys. Rev. Lett. 113, 106802 (2014).

[47] T. Low, A. S. Rodin, A. Carvalho, Y. Jiang, H. Wang, F. Xia, and A. H. Castro Neto, Phys. Rev. B 90, 075434 (2014).

[48] F. Cadiz, D. Paget, and A. C. H. Rowe, Phys. Rev. Lett. 111, 246601 (2013).

[49] W. Ebeling, D. Blaschke, R. Redmer, H. Reinholz, and G. Rpke, J. Phys. A: Math. Theor. 42, 214033 (2009).

[50] H. J. Krenner, E. C. Clark, T. Nakaoka, M. Bichler, C. Scheurer, G. Abstreiter, and J. J. Finley, Phys. Rev. Lett. 97, 076403 (2006). 